\title{
Intra-articular pressure profile of the knee joint in a spectrum of inflammatory arthropathies
}

\author{
Shahid Jawed, Karl Gaffney, David R Blake
}

\begin{abstract}
Objectives-The intra-articular pressure (IAP) rises significantly after isometric quadriceps contraction in patients with rheumatoid synovitis, a process that may temporarily impede synovial blood flow and cause oxidative injury. In acute traumatic knee effusions (ATE) pressure rises are trivial. This study compared the IAP profiles of patients with ATE with three different populations-an acute synovitis on the background of a chronic inflammatory arthropathy, a chronic low grade inflammatory arthropathy, and an acute intermittent inflammatory arthropathy. The study objective was to discover if the pressure profiles observed in these groups reflect an influence of the inflammatory process or time or both.
\end{abstract}

Methods-Thirty three patients were studied. These were divided into four subgroups; group 1: five acute traumatic knee effusions (ATE); group 2: acute effusions on the background of a chronic inflammatory arthropathy: seven rheumatoid arthritis (RA), five psoriatic arthritis (PsA); group 3: seven osteoarthritis (OA) and group 4: acute effusions on the background of an intermittent inflammatory arthropathy: seven pyrophosphate arthropathy (PA), one amyloid (AA), one Behcet's (B). IAP was measured ( $\mathrm{mm}$ Hg) at rest and during isometric quadriceps contraction using the hand held portable 295-1 intra-compartmental pressure monitor system (Stryker UK). The volume of synovial fluid aspirated was recorded.

Results-Expressed as medians (interquartile range). Resting IAP was; ATE 6 (2-12), RA 8 (5-47), PsA 18 (11-31), OA 17 (7-21), PA 25 (9-29), AA 14, and B 12. IAP increased in all subjects during isometric contraction; ATE 9 (7-16), RA 56 (33-150), PsA 52 (43-85), OA 56 (20-116), PA 53 (4165), AA 47, B 57 and the IAP rise was significant $(p<0.05)$ in all except the ATE group $(p>0.05)$. The volume of synovial fluid aspirated in groups 2,3 , and 4 correlated significantly with the magnitude of the IAP change $(r=0.45, p<0.05)$.

Conclusion-The IAP rise during isometric quadriceps contraction is a feature of all patients with an inflammatory based effusion irrespective of the duration of the effusion. This is not the case in patients with an ATE. In inflammatory synovitis the rise in intra-articular pressure with isometric quadriceps contraction relates to effusion volume. It is concluded that the inflammatory process prevents reflex muscle inhibition, a locally protective mechanism that minimises the potential for intermittent ischaemia/oxidative injury.

(Ann Rheum Dis 1997;56:686-689)

Many inflammatory joint disorders manifest as a persistent synovitis. Many mechanisms have been proposed to explain why inflammatory arthritis is so peculiarly persistent. One such explanation centres on oxidative injury secondary to intermittent ischaemia induced by movement 'hypoxic-reperfusion injury'. ${ }^{1}$ The normal human synovial joint has a subatmospheric or low atmospheric intra-articular pressure (IAP) at rest and during exercise. ${ }^{23}$ In chronically inflamed rheumatoid joints, the resting IAP is increased and rises further during exercise. This pressure rise on exercise may exceed capillary perfusion pressure (CPP). Even a rise in IAP of 10-30 $\mathrm{mm} \mathrm{Hg}$ can compromise capillary perfusion in supine rheumatoid arthritis (RA) patients. ${ }^{4}$ After cessation of exercise, capillary blood flow returns, often to suprabasal levels, restoring tissue blood supply. This sequence of repetitive but transient ischaemic episodes, each followed by reperfusion of the synovium has the capacity to generate tissue damaging reactive oxygen species by uncoupling a series of intracellular redox systems. Studies of diseased human synovium subjected to 'hypoxic-reperfusion cycles' ex-vivo show generation of reactive oxygen species $^{5}$ and studies of synovial fluid after both exercise cycles or isometric quadriceps contraction (IQC) show evidence of oxidant damage to a variety of biomolecules including proteins, lipids, lipoproteins, and carbohydrates with depletion of antioxidants locally within the joint. ${ }^{6}$

In a previous study we compared the IAP profile of patients with RA and chronic knee effusions with normal subjects with acute self limiting traumatic effusions. ${ }^{7}$ Patients with a traumatic synovitis, although they have a raised resting IAP, in contrast with the RA patients, do not experience a significant pressure rise with exercise. In this study we have investigated the pressure response to IQC in four groups of patients. The first group were patients with an acute traumatic knee effusion with no previous arthropathy. The second group were patients with a chronic inflammatory arthropathy having an acute flare of their knee synovitis. The third group were patients with osteoarthritis 
(OA) associated with recurrent episodes of knee effusions without any evidence of any other inflammatory process. The fourth group were patients with an intermittent inflammatory arthropathy who currently had an acutely inflamed knee but had no evidence of synovitis between episodes. The question we posed was-Do patients with a chronic inflammatory arthropathy, chronic inflammatory OA or an intermittent inflammatory arthropathy with a superimposed acute synovitis adopt a pressure profile in their acutely inflamed joint typical of an acute traumatic effusion; or do they develop a profile, as seen in those with chronic RA, which provides a pressure profile conducive to cycles of hypoxic reperfusion injury ? The question allows us to determine if time or the inflammatory process are the major influences on whether the joint sustains significant pressure responses.

\section{Methods}

After ethics committee approval of the study protocol, 33 clinically well defined patients with acute knee effusions were recruited to the study over a period of one year. The median patient age was 26 years (16-54) for the acute traumatic effusions (ATE, group 1), 59.5 years (32-78) for the chronic inflammatory arthropathies (group 2), 73 years (65-82) for the OA (group 3), and 70 years (43-82) for the acute intermittent inflammatory arthropathies (group 4). Group 1 contained five patients who were previously well and had presented within 48 hours of sustaining an ATE, group 2 contained 12 patients (seven RA and five psoriatic arthritis (PsA)), group 3 contained seven patients with $\mathrm{OA}$, and group 4 contained nine patients (seven with pyrophosphate arthropathy (PA), one Behcet's (B), and one amyloid (AA)). The patients in groups 2,3 , and 4 were seen as soon as possible after the onset of their knee effusions (0-3 weeks). At presentation patients were entered into the study if their clinical diagnosis was not in dispute on examination (pain and local tenderness; a joint effusion with a positive bulge sign or positive patellar tap). Our patients in groups 2,3 , and 4 had all suffered previous episodes of knee synovitis. Informed consent was obtained from all participants. IAP was measured $(\mathrm{mm} \mathrm{Hg}$ ) using the hand held portable 295-1 intracompartmental pressure monitor system (Stryker UK). A digital multimeter (ISOTECH IDM 63) was attached to the pressure monitor system to determine whether recorded pressures were atmospheric or subatmospheric.

The details of the system are reported elsewhere ${ }^{8}$ but briefly the pressure monitor system consists of two principal components; a digital monitor containing a solid state transducer and case with auto zeroing device, and a sterile disposable pressure monitor set containing a pre-filled saline syringe and diaphragm chamber to maintain a sterile fluid pathway. After assembly, the pressure monitor set is fitted with a 21 gauge needle. The system was calibrated with the BIO-TEC electronic cali- brator and by a water manometer according to the manufacturer's instructions.

Patients were rested in a supine position for at least 15 minutes before the procedure. Subcutaneous tissues were infiltrated with $2 \%$ lignocaine taking care to avoid capsular or intra-articular contact. The pressure monitor set was assembled and primed by slowly forcing saline through the chamber to the needle tip to ensure hydrodynamic communication with the joint lumen. The monitor was then set at zero and the needle introduced into the joint cavity (lateral approach). Accurate intra-articular placement was supported by aspiration of synovial fluid after IAP measurement. IAP was measured at rest and after repeated maximal IQC against resistance. At least three cycles were recorded in each person and the mean value reported. After IAP measurement, joints were aspirated to dryness and the volume of synovial fluid recorded. The pressure response was related to effusion volume in each of the four groups studied.

\section{STATISTICAL ANALYSIS}

Data were not normally distributed; nonparametric statistical methods were applied. Resting and quadriceps contraction induced changes were compared using the Wilcoxon signed rank test. The Mann-Whitney $U$ test was applied to assess the IAP differences between patient groups. The relation between volume of synovial fluid aspirated and the magnitude of the exercise induced IAP rise was assessed using Spearman's rank correlation coefficient.

\section{Results}

IAP AT REST AND DURING EXERCISE

All results are quoted as medians (interquartile range). Figure 1 shows IAP at rest and during IQC in respective patient groups. Resting IAP $(\mathrm{mm} \mathrm{Hg})$ were in group 1; ATE 6 (2-12), group 2; RA 8 (5-47), PsA 18 (11-31): group 3; OA 17 (7-21), and group 4; PA 25 (9-29), B 12, AA 14. During IQC, the IAP (range) increased significantly $(\mathrm{p}<0.05)$ in groups 2,3 , and 4 but not in group 1 . The exercise induced values were; group 1; ATE 9 (7-16), group 2; RA 56 (33-150), PsA 52 (43-85): group 3; OA 56 (20-116), and group 4; PA 53 (41-65), B 57, AA 47 . The rise in IAP in groups 2,3 , and 4 was significantly higher than that seen in group $1(\mathrm{p}<0.05)$. Resting and exercise related IAP values seemed unrelated to either aetiology, total disease duration or duration of the acute episode.

\section{Influence of joint aspiration and synovial fluid} volume on IAP generation

Synovial fluid was aspirated from all patients (median (IQR)): group 1; $18 \mathrm{ml}$ (5-33), group $2 ; 21.5 \mathrm{ml}$ (3-55), group 3; $15 \mathrm{ml}$ (9-40), and group $4 ; 8 \mathrm{ml}(3-50)$. There was a significant correlation between volume of synovial fluid aspirated and the magnitude of the exercise related IAP rise in groups 2,3 , and 4 when combined $(r=0.45, \mathrm{p}<0.05)$. No such relation exists in group $1(r=0.21, \mathrm{p}=0.74)$. Figure 2 illustrates these results. 


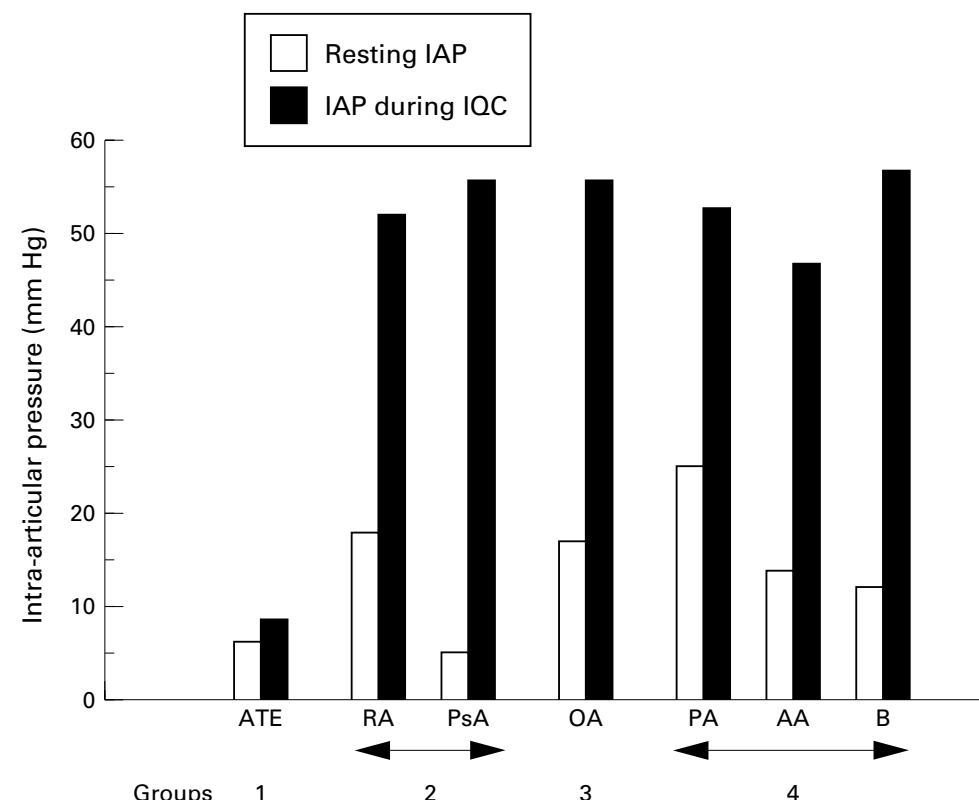

Figure 1 IAP at rest and during exercise shows the differences between resting IAP and IAP during IQC; group 1: ATE; group 2: RA and Ps A; group 3: $O A$; group 4: $P A, B$, and $A A$. disease process is the key factor in determining the IAP profile and not the duration of the effusion.

In previous studies of IAP at rest and during exercise, we and others have shown that normal peripheral synovial joints have a subatmospheric pressure, commonly -2 to $-4 \mathrm{~mm} \mathrm{Hg}$ at rest. ${ }^{13}$ This pressure falls with exercise or during IQC. ${ }^{2}$ Two teleological explanations have been offered for this finding. The first is that cartilage is avascular because of the compressive forces it sustains, and its nutrition is dependent on synovial vessel perfusion. ${ }^{9}$ Maintenance of synovial patency by mild distraction, a consequence of increased muscle tone, assists cartilage nutrition despite exercise or compressive forces. It has also been proposed that negative pressure will draw loose synovial tissues towards each other during exercise, thus offering a stabilising influence and promoting normal joint tracking. ${ }^{10}$

In acute traumatic synovitis the situation is fundamentally different. The resting IAP is increased and though rising a little on exercise, does not exceed the capillary perfusion pressure. ${ }^{4}$ The probable explanation for this is that an acute effusion invokes reflex muscle inhibition (RMI). This hypothesis is supported by previous electromyographic (EMG) studies demonstrating RMI in acutely effused knees. ${ }^{11}{ }^{12}$ It is suggested that stimulation of capsular or intra-articular receptors in an acute knee effusion inhibits the lower motor neurone response to the quadriceps muscle thus preventing contraction. This process of RMI, we suggest, is beneficial, and the loss of the Hoffman reflex prevents a traumatic effusion compromising synovial blood flow. In this study we show that patients with an acute effusion on the background of a chronic inflammatory arthropathy, chronic OA or an acute intermittent inflammatory arthropathy, lose what we would suggest is a defence system. Failure of RMI seems to occur within a few days of developing an acute inflammatory reaction; although from this cross sectional study, we are unable to define the precise time of onset with greater precision.

We would speculate as to the explanation. One feature that differentiates an acute traumatic effusion from an acute inflammatory response is the nature of the infiltrating cellular reaction. Both activated neutrophils and macrophages are a feature of the latter, and not present to any significant extent in the former. Both cell types release a variety of oxidants, which singularly or in combinations have the capacity to damage both biomolecules and articular structures. ${ }^{13}$ Animal model studies have demonstrated that nerves of the normal synovium are damaged after a brief exposure to oxidant injury. ${ }^{14}$ Studies of the inflamed rheumatoid synovium have shown a similar pattern of neuronal loss. ${ }^{15}$

In support of this hypothesis is a clinical study by de Andrade et al. ${ }^{16}$ In this study quadriceps RMI was measured clinically and by EMG (in some patients) in a series of normal volunteers and patients with various knee complaints whose knee joints were injected with 
plasma. The results showed that distension of the knee joint with plasma resulted in weakening of the quadriceps muscle in 16 subjects. This protective mechanism of RMI was lost in a patient with neuropathic disease (Charcot joint) and was delayed in other subjects where normal joints were first instilled with intraarticular local anaesthetic and then injected with plasma. This study suggests that stimuli from the knee joint reflexly inhibit the lower motor neurones supplying the quadriceps muscle. From the results of this study it would seem that the intra-articular innervation plays a key part in sustaining RMI, a hypothesis that we would support.

These results lead us to hypothesise that the rise in IAP seen in groups 2,3 , and 4 is conducive to causing cycles of hypoxic reperfusion injury in patients with inflammatory effusions. This of course assumes that our model of IQC in the supine patient accurately reflects the situation in walking patients. In the upright position the capillary perfusion pressure may be higher than expected because of the additional effects of gravity. The exact capillary perfusion pressure in the walking patient with an inflamed knee is not known but even allowing for a higher capillary perfusion pressure in the upright position, the IAPs generated during IQC (up to $178 \mathrm{~mm}$ $\mathrm{Hg}$ ) would still be sufficient to obstruct blood flow temporarily. Geborek et $a l^{4}$ showed clearly in their study that an increase in IAP of as little as $20 \mathrm{~mm} \mathrm{Hg}$ in patients with effused knees can significantly decrease synovial blood flow suggesting the joint to be a system with a blood flow that is very sensitive to comparatively small changes in IAP.

KG and SJ are ARC Clinical Research Fellows; DRB is an ARC Professor of Rheumatology. We would like to thank Dr David Walsh, Dr Paul Mapp, and Dr Ian Chikanza for their helpful comments in preparing this manuscript.
1 Blake DR, Merry P, Unsworth J, Kidd BL, Outhwaite J, Ballard R, et al. Hypoxic-reperfusion injury in the inflamed human joint. Lancet 1989;i:289-93.

2 Baxendale RH, Ferrell WR, Wood L. Intra-articular pressure during active and passive movement of normal and distended human knees. J Physiol 1985;369:179P.

3 Jayson MIV, Dixon A St J. Intra-articular pressure in rheumatoid arthritis of the knee. III Pressure changes during joint use. Ann Rheum Dis 1970;29:401-8.

4 Geborek P, Forslind K, Wollheim FA. Direct assessment of synovial blood flow and its relation to induced hydrostatic pressure changes. Ann Rheum Dis 1989;48:281-6.

5 Singh D, Nahzat NB, Fairburn K, Sahinoglu T, Blake DR, Jones P. Electron spin resonance spectroscopic demonstration of reactive oxygen species by diseased synovial tissue following ex-vivo hypoxia-reoxygenation. Ann Rheum Dis 1995;54:94-9.

6 Fairburn K, Stevens CR, Winyard PG, Kus M, Ward RJ, Cunningham J, et al. Oxidative stress and its control: A pathogenetic role in inflammatory joint disease. Biochem Soc Trans 1993; 21:371-5.

7 Merry P, Williams R, Cox N, Blake DR. Comparative study of intra-articular pressure dynamics in joints with acute traumatic and chronic inflammatory effusions: potential implications for hypoxic-reperfusion injury. Ann Rheum Dis 1991;50:917-20.

8 Gaffney K, Williams RB, Jolliffe VA, Blake DR. Intraarticular pressure changes in rheumatoid and normal peripheral joints. Ann Rheum Dis 1995;54:611-2.

9 Simkin PA, Bassett JE, Koh EM. Synovial perfusion in the human knee: a methodologic analysis. Semin Arthritis Rheum 1995;25:56-66.

10 Simkin PA. Feeling the pressure. Ann Rheum Dis 1995;54: 611-3.

11 Stratford P. Electromyography of the quadriceps femoris muscles in subjects with normal knees and acutely effused knees. Phys Ther 1981;3:279-83.

12 Spencer JD, Hayes KC, Alexander IJ. Knee joint effusion and quadriceps reflex inhibition in man. Arch Phys Med Rehabil 1984;65:171-7.

13 Palmer DG, Hogg N, Revell PA. Lymphocytes, polymorphonuclear leukocytes, macrophages and platelets in synovium involved by rheumatoid arthritis. A study with monoclonal antibodies. Pathology 1986;18:431-7.

14 Mapp PI, Walsh DA, Garrett NE, Kidd BL, Cruwys SC, Polak JM, et al. Effect of three animal models of inflammation on nerve fibres in the synovium. Ann Rheum Dis 1994;53:240-6.

15 Mapp PI, Kidd BL, Gibson SJ, Terry JM, Revell PA, Ibrahim NB, et al. Substance P-, calcitonin gene-related peptide- and C-flanking peptide of neuropeptide Y-immunoreactive fibres are present in normal synovium but depleted in patients with rheumatoid arthritis. Neuroscience 1990;37:143-53.

16 De Andrade JR, Grant C, Dixon A StJ. Joint distension and reflex muscle inhibition in the knee. J Bone Joint Surg 1965;47:313-22. 